\title{
Lung retransplantation in the modern era
}

\author{
A. Justin Rucker, Joseph R. Nellis, Jacob A. Klapper, Matthew G. Hartwig \\ Division of Cardiovascular and Thoracic Surgery, Department of Surgery, Duke University Medical Center, Durham, NC, USA \\ Contributions: (I) Conception and design: None; (II) Administrative support: None; (III) Provision of study materials or patients: None; (IV) \\ Collection and assembly of data: None; (V) Data analysis and interpretation: None; (VI) Manuscript writing: All authors; (VII) Final approval of \\ manuscript: All authors. \\ Correspondence to: A. Justin Rucker, MD, MPH. Box 3531, Duke University Medical Center, 2301 Erwin Road, Durham, NC 27710, USA. \\ Email: alvin.rucker@duke.edu.
}

\begin{abstract}
Chronic lung allograft dysfunction remains the leading cause of long-term morbidity and mortality for lung transplant recipients. Lung retransplantation currently represents the only therapeutic option for patients for refractory allograft dysfunction. However, debate remains regarding both the efficacy and ethicality of lung retransplantation in light of the shortage of lung allografts. The aim of this review is to discuss the available literature on lung retransplantation in the current era. Through this we hope to provide insight into ideal patient selection, donor organ selection, surgical approaches, and future considerations within the field in order to improve outcomes and best address organ utilization while a waitlist continues to exist. Lung retransplantation in select patients can offer comparable survival outcomes to primary lung transplantation. However, several risk factors including retransplantation with the first year of primary transplantation, older age, poor functional status, and ICU level requirements prior to transplantation are associated with worsened outcomes. Donor organ selection considerations are comparable to those in primary lung transplantation. However, surgical approach is often impacted by dense pleural and mediastinal adhesions in the recipient which increase the complexity of the hilar dissection. The postoperative course is often more complex for patients undergoing retransplantation compared to those undergoing primary lung transplant as well. However, pending more data on long term outcomes in lung retransplantation and the potential impact of retransplant recipients on waitlist mortality, lung retransplantation should remain in use primarily for the treatment of chronic graft dysfunction in carefully selected patients.
\end{abstract}

Keywords: Lung transplantation; lung retransplantation; chronic lung allograft dysfunction (CLAD)

Submitted Mar 23, 2021. Accepted for publication Jun 22, 2021.

doi: $10.21037 /$ jtd-2021-25

View this article at: https://dx.doi.org/10.21037/jtd-2021-25

\section{Introduction}

Lung transplantation is the standard of care for end-stage lung disease. Since 1990, over 70,000 lung transplants have been performed worldwide with over 4,500 in 2017 alone (1). Despite advances in the field and the growing number of transplants performed annually, acute and chronic allograft dysfunction continue to be the leading causes of morbidity and mortality in this population, limiting median graft survival to 6.2 years (1-5). For a select group of patients, repeat lung transplantation is an option for refractory graft dysfunction. Since 2000, lung retransplantation represents $3-5 \%$ of the annual lung transplant volume (1). As the number of primary lung transplants has grown nearly three-fold over the past twenty years, so too has the number of patients undergoing retransplantation which now approaches close to 200 cases per year. The following article summarizes the available literature on lung retransplantation, including patient selection, donor organ selection, surgical approaches, and future considerations within the field, in order to improve outcomes and best address organ utilization while a waitlist continues to exist. 


\section{Patient selection}

Patients with medically refractory allograft dysfunction are candidates for lung retransplantation, although the decision to list patients for retransplantation is ultimately left to the individual transplant center. Historically, there was stigma associated with relisting patients as lungs were allocated based on total waitlist time which allowed some patients to be retransplanted while others were dying before they could receive their first allograft (6,7). By 2005, the United Network for Organ Sharing (UNOS) implemented the Lung Allocation Score (LAS) which objectively profiled patients based on current medical urgency and expected survival measured at one year in order to decrease waitlist mortality and more equitably distribute lungs to those in need. Median wait time fell from two years to 200 days and waitlist mortality was nearly halved as lungs were more appropriately distributed based on the severity of patients' underlying diagnoses $(6,8)$. A consequence of the LAS was that priority was also given to patients who were relisted for transplantation as the waitlist mortality for chronic lung allograft dysfunction (CLAD) and idiopathic pulmonary fibrosis are similar $(6,9,10)$. Despite being given priority, the percentage of patients undergoing lung retransplantation per year has remained stable in recent years. This trend likely reflects center specific decisions to limit retransplantation due to increased surgical complexity (11).

Some analyses suggest that long-term graft survival is worse with lung retransplantation. In a 2017 review of their outcomes between 2008 and 2015, the Organ Procurement and Transplantation Network demonstrated inferior 1,3 , and 5 years survival for patients undergoing lung retransplantation $(76.0 \%, 48.9 \%$, and $33.8 \%)$ relative to those undergoing their first transplantation $(87.9 \%, 70.5 \%$, and $56.0 \%)(12)$. However, after adjusting for comorbidities, minimal differences in survival are observed between primary and repeat lung transplantation (10). Risk factors associated with worse outcomes following retransplantation include early retransplantation within the first year, older age, female gender, 6-minute walk test less than 400 feet, donor-recipient height ratio $<1$, poor functional status, ICU level requirements prior to transplantation, operations performed at lower volume transplant centers, intravenous antibiotics within 2 weeks of transplantation, and single lung rather than double lung retransplantation (13-16). Careful selection of recipients for retransplantation based on known or yet to be determined risk factors will lead to overall better survival for this group of patients.
Patient selection becomes increasingly important when physicians are asked to offer bridging therapy prior to lung retransplantation. Mechanical ventilation and, more recently, extracorporeal membrane oxygenation (ECMO) are readily available at transplant centers. However, there is a paucity of literature on the subject of retransplantation in mechanically ventilated patients (10,17-19). Although many of these data could be considered somewhat dated, it is clear that retransplant candidates on mechanical ventilatory support are at high risk for inferior outcomes. Those requiring bridging ECMO prior to retransplantation are typically in even worse preoperative condition than those requiring mechanical ventilatory support. Of the few single center reviews on this subject, these patients uniformly have more complicated intraoperative and perioperative courses (20-22). A meta-analysis of the cumulative experience points to improved survival for those with awake, bridging venovenous (VV) rather than venoarterial (VA) ECMO at 1 year (67\% vs. $20 \%$ ), although results continue to be limited by small sample sizes and influenced by institutional experience (21). At our institution, we are extremely selective about who we bridge to retransplantation given these realities.

The most common indication for patients undergoing lung retransplantation is CLAD. Several recent single institution series have demonstrated comparable survival despite more complex perioperative courses for patients who receive retransplantation for CLAD compared to those that undergo primary lung transplantation, although the results of these studies are highly subject to selection bias $(20,23,24)$. Successful of retransplantation for CLAD appears to be at least partially contingent on the underlying CLAD phenotype. First described by Sato et al. in 2011, restrictive allograft syndrome (RAS) is physiologically different than bronchiolitis obliterans syndrome (BOS), the more common form of chronic lung allograft dysfunction (25). Patients with RAS have worse prognosis than those with BOS, and this includes outcomes following lung retransplantation (21). In a 2015 study by Verleden et al., patients undergoing lung retransplantation for RAS redeveloped CLAD sooner and ultimately had worse survival than patients with BOS. Survival for patients with RAS at 1, 3, and 5 years after retransplantation was $59 \%, 33 \%$, an $28 \%$ with a median graft survival of 1.7 years, whereas patients with BOS experienced survival of $84 \%, 67 \%$, and $51 \%$ with a median graft survival of 5.1 years (22).

The careful selection of patients for lung retransplantation is crucial for successful outcomes given the complexity 
in operative and perioperative management. Patients who stand to benefit the most from retransplantation are those who have experienced reasonable survival following their initial transplantation and are not suffering from additional organ dysfunction (i.e., kidney, liver). In addition, it is important to consider the subtype of CLAD they are experiencing as this appears to impact outcomes. In general, retransplantation in patients who are mechanically ventilated or on ECMO prior to transplantation should only be used very selectively.

\section{Donor organ selection}

Like primary lung transplantation, the assessment of donor lungs for retransplantation should take into consideration the relative size of the lungs, donor specific antibodies (DSA), and the risk of waitlist mortality. For patients undergoing primary lung transplantation, undersized lungs have been associated with an increased occurrence of primary graft dysfunction, CLAD, and mortality at 1 and 5 years for all comers, although outcomes may be dependent on underlying diagnosis (26-29). Available methods based on height, gender and height, chest X-ray, and thoracic perimeter have all been shown to carry significant variability (30-32). No studies to date have been done to specifically assess the impact of lung size mismatch in lung retransplantation. In our experience, the chest cavity is more rigid and fixed than in primary lung transplantation, which may adversely impact oversized lungs Therefore, an emphasis should be placed on accurately matching donorrecipient size in lung retransplantation.

In addition to pregnancy and blood transfusions, prior transplantation is a risk factor for sensitization, high levels of HLA antibodies, and difficulty finding a compatible organ, especially for multiparous women (29). In general, patients with higher cPRAs experience longer waitlist time, decreased rate of transplantation, and increased waitlist mortality than their peers with lower cPRAs. However, following transplantation, patients with high pretransplant DSAs and those who develop persistent de novo DSAs in the posttransplant setting have higher rates of antibody mediated rejection, CLAD, and overall mortality $(33,34)$. Therefore, if a retransplant candidate screens positive for having DSAs to their prior donor, those HLA antigens should be avoided when matching a subsequent donor. This can be done prior to transplant with a virtual or prospective crossmatch. Although induction therapies exist for patients with high cPRAs including intraoperative IVIG and plasmapheresis with antithymocyte globulin and rituximab, no randomized trials have been performed to standardize protocols in these patients and we have little data to guide our decision making $(35,36)$.

\section{Surgical approaches and expectations}

There are multiple factors to consider when deciding an optimal operative approach in retransplantation. One such factor is whether a single or bilateral orthotopic lung transplantation (BOLT) is more appropriate. In an analysis of Scientific Registry of Transplant Recipients (SRTR) data, Kon and colleagues examined outcomes for retransplantation for patients who had undergone a previous single lung transplant. They found that patients who received a contralateral single lung retransplants had similar survival at 30 days, 1 year, and 5 years to those that received bilateral retransplants, but that survival for both were superior to that of patients who received ipsilateral single lung transplant (37). Clearly, from a technical standpoint avoiding the original site of transplant and transplanting the contralateral lung when possible should be easier. However, one must ensure this is medically appropriate (i.e., no residual infections, etc.) and technically feasible.

The incisions in patients undergoing re-transplant, whether it be a SOLT or BOLT, are no different than it was at the time of initial transplant (35). The predominant patient population that we consider for re-transplant are individuals who have had a previous BOLT. However, this is mainly because BOLTs are more commonly performed for primary lung transplantation. Also, at many centers SOLTs are reserved for older, more debilitated patients. At the time of retransplantation, it is expected that the patients will have dense pleural and mediastinal adhesions, and we are meticulous in attempting to lyse these with minimal bleeding. Once this is accomplished, our next goal is to obtain central control of the pulmonary arteries (PA) within the pericardium as dissection at the level of the hilum is treacherous given the adhesions between the prior pulmonary anastomosis and the bronchus. If this can be successfully accomplished, we generally cannulate centrally for VA ECMO as it requires less heparinization and therefore in theory will mean less blood product transfusions. Occasionally if central PA control is not possible, full cardiopulmonary bypass (CPB) is initiated, and the main PA is cannulated which decompresses the pulmonary system and allows for the subsequent division of the prior anastomoses within the hilum. Some surgeons 
at the time or primary transplantation will cover their bronchial anastomoses with peribronchial tissues or pedicled thymic flaps. These techniques make it more likely that the bronchus and PA can be separated at the subsequent transplantation.

Similar to primary lung transplantation, less invasive strategies for retransplantation have also been explored. Sommers et al. published a series which analyzed their experience with lung retransplantations after transitioning to less invasive transplant protocols which included performing retransplant via minimally invasive, sternumsparing anterolateral thoracotomies without the use of $\mathrm{CPB}$ or ECMO and without heparin administration. These patients were compared to a historical cohort of patients who underwent retransplantation prior to the adaptation of these protocols. They found that while operative times were longer, time to extubation and length of ICU stay as well as survival at 30 days and 1 year were improved in the era of less invasive lung retransplantation (38). More recently, this group has also transitioned to performing lung transplantations on ECMO in appropriate candidate and have demonstrated comparable survival despite higher perioperative risk in the patients who received transplant on ECMO (39). While use of a historical control is a source of potential bias in the earlier study, their results do suggest a sternal sparing approach is at least technically possible in select cases of lung retransplantation. However, the overall safety and benefit beyond the traditional approach remains to be clearly demonstrated. Again, one technical suggestion to increase the chances of a more minimally invasive dissection at the time of retransplantation is to separate the hilar PA from surrounding structures with tissue (i.e., peribronchial tissue or a thymic pedicle).

Following successful surgery, patients undergoing lung retransplantation continue to have a difficult postoperative course. Halloran and colleagues found that compared to patients that underwent primary lung transplantation, those that received retransplantation had increased postoperative days on the ventilator and, consequently, increased ICU length of stay (23). However, in other series, postoperative ventilator time was comparable between primary transplant and retransplant patients $(20,40)$. Postoperatively, patients report similar improvements in their quality of life after retransplantation as they did following their primary transplant. Although despite these improvements, twenty five percent of the patients who required complete assistance with their activities of daily living preoperatively will continue to require maximum assistance postoperatively (15).
With respect to their infectious risk, patients undergoing retransplantation do not experience increased rates of graft colonization or infection compared to those undergoing primary lung transplantation. Ultimately, however, similar to primary transplants, patients undergoing retransplantation typically die as a consequence of graft failure or infectious complications (23).

\section{Future directions}

While the current literature would suggest that lung retransplantation can offer comparable outcomes to primary transplantation in select patients, there remains debate regarding the ethical implications of repeat transplantation (7). This debate was instigated by long waitlist times, high waitlist mortality, and perceived inequity in the lung allocation process, particularly for those waiting for their first transplant. Although after the implementation of LAS in 2005, waitlist time decreased by over $70 \%$ and mortality by nearly $50 \%(6,8)$. The LAS, as previously described, attempts to maximize the utility of lungs, and can be thought of as the difference between predicted near-term and 1-year mortality for patients on the waitlist. These improvements settled many concerns about fairness in organ allocation, although interestingly, they also helped patients undergoing retransplantation receive new allografts sooner $(6,9,10)$. Despite not having as high of 1-year predicted survival as a primary transplant candidate, retransplant candidates benefit from the LAS preferentially allocating organs to patients with higher predicated waitlist mortality. Despite the improvement in waitlist mortality and simultaneous improvement in access to retransplantation, the percentage of patients undergoing lung retransplantation has remained relatively stable over time. Without regulatory guidelines restricting patients from being listed, the stability in retransplantation rates is likely attributable to transplant center specific decisions and more modern ethical debate around resource utilization and expected quality of life. Lung retransplantation represents a thin line between hope and false expectations, and this can be difficult for some patients and providers to understand or accept (7). There is a lack of evidence on these topics within lung retransplantation, making the decision to list someone for retransplantation more experiential than empirical at times for physicians. To help with this decision, other specialties have established minimum requirements for long-term survival (41). While this does not yet currently exist in lung transplantation, it remains unclear 
whether that would be a step forward or backwards for the community. Providers appear to understand this complex interplay as represented by stable numbers of retransplants. The ethical concerns further emphasize the importance of patient selection in this patient population.

\section{Conclusions}

Long term efficacy of lung transplantation continues to be challenged by CLAD. At this time, lung retransplantation represents the only viable therapeutic option in this context and can demonstrate similar efficacy to primary lung transplantation in select patients. While previous studies highlight the feasibility of lung retransplantation, further work is needed to clarify its impact on broader waitlist mortality. Moreover, future studies will be necessary to explore how the possible effects of lung retransplantation on waitlist mortality might be mitigated through advances in efforts to expand the donor pool as well as those aimed to extend the longevity of the primary allografts. Therefore, technical, medical and ethical concerns continue to be delineated and discussed and lung retransplantation should remain in use primarily for the treatment of chronic graft dysfunction in carefully selected patients.

\section{Acknowledgments}

Funding: None.

\section{Footnote}

Provenance and Peer Review: This article was commissioned by the Guest Editor (Jonathan D'Cunha) for the series "Lung Transplantation: Past, Present, and Future" published in Fournal of Thoracic Disease. The article has undergone external peer review.

Conflicts of Interest: All authors have completed the ICMJE uniform disclosure form (available at: https:// dx.doi.org/10.21037/jtd-2021-25). The series "Lung Transplantation: Past, Present, and Future" was commissioned by the editorial office without any funding or sponsorship. MGH reports and Research and/or consulting for Mallinkrodt, Biomedinnovations, Paragonix in the area of lung transplantation. All authors have no other conflicts of interest to declare.

Ethical Statement: All authors are accountable for all aspects of the work in ensuring that questions related to the accuracy or integrity of any part of the work are appropriately investigated and resolved.

Open Access Statement: This is an Open Access article distributed in accordance with the Creative Commons Attribution-NonCommercial-NoDerivs 4.0 International License (CC BY-NC-ND 4.0), which permits the noncommercial replication and distribution of the article with the strict proviso that no changes or edits are made and the original work is properly cited (including links to both the formal publication through the relevant DOI and the license). See: https://creativecommons.org/licenses/by-nc-nd/4.0/.

\section{References}

1. The International Society for Heart and Lung Transplantation. The International Thoracic Organ Transplant (TTX) Registry of the International Society for Heart and Lung Transplantation: Thirty-Sixth Annual Report. J Heart Lung Transplant 2019;38:1015-66.

2. Snell GI, Levvey BJ, Levin K, et al. Donation after Brain Death versus Donation after Circulatory Death: Lung Donor Management Issues. Semin Respir Crit Care Med 2018;39:138-47.

3. Wierup P, Haraldsson A, Nilsson F, et al. Ex vivo evaluation of nonacceptable donor lungs. Ann Thorac Surg 2006;81:460-6.

4. Opelz G, Döhler B, Ruhenstroth A, et al. The collaborative transplant study registry. Transplant Rev (Orlando) 2013;27:43-5.

5. Chambers DC, Yusen RD, Cherikh WS, et al. The Registry of the International Society for Heart and Lung Transplantation: Thirty-fourth Adult Lung And HeartLung Transplantation Report-2017; Focus Theme: Allograft ischemic time. J Heart Lung Transplant 2017;36:1047-59.

6. Eberlein M, Garrity ER, Orens JB. Lung allocation in the United States. Clin Chest Med 2011;32:213-22.

7. Godinas L, Van Raemdonck D, Ceulemans LJ, et al. Lung retransplantation: walking a thin line between hope and false expectations. J Thorac Dis 2019;11:E200-3.

8. Yusen RD, Shearon TH, Qian Y, et al. Lung transplantation in the United States, 1999-2008. Am J Transplant 2010;10:1047-68.

9. Egan TM, Murray S, Bustami RT, et al. Development of the new lung allocation system in the United States. Am J Transplant 2006;6:1212-27. 
10. Kawut SM, Lederer DJ, Keshavjee S, et al. Outcomes after lung retransplantation in the modern era. Am J Respir Crit Care Med 2008;177:114-20.

11. Weill D, Benden C, Corris PA, et al. A consensus document for the selection of lung transplant candidates: 2014--an update from the Pulmonary Transplantation Council of the International Society for Heart and Lung Transplantation. J Heart Lung Transplant 2015;34:1-15.

12. Organ Procurement and Transplantation Network. Lung kaplan-meier patient survival rates for transplants performed: 2008-2015. 2017. https://optn.transplant.hrsa. gov/data/view-data-reports/national-data/\#. Accessed December 192017.

13. Clausen ES, Weber JM, Neely ML, et al. Predicting survival for lung retransplantation patients at one and five years. J Heart Lung Transplant 2019;38:S225-S6.

14. Ren D, Kaleekal TS, Graviss EA, et al. Retransplantation Outcomes at a Large Lung Transplantation Program. Transplant Direct 2018;4:e404.

15. Kilic A, Beaty CA, Merlo CA, et al. Functional status is highly predictive of outcomes after redo lung transplantation: an analysis of 390 cases in the modern era. Ann Thorac Surg 2013;96:1804-11; discussion 11.

16. Osho AA, Castleberry AW, Snyder LD, et al. Differential outcomes with early and late repeat transplantation in the era of the lung allocation score. Ann Thorac Surg 2014;98:1914-20; discussion 20-1.

17. Novick RJ, Stitt LW, Al-Kattan K, et al. Pulmonary retransplantation: predictors of graft function and survival in 230 patients. Pulmonary Retransplant Registry. Ann Thorac Surg 1998;65:227-34.

18. Hall DJ, Belli EV, Gregg JA, et al. Two Decades of Lung Retransplantation: A Single-Center Experience. Ann Thorac Surg 2017;103:1076-83.

19. Yang J, Pan X, Fu S, et al. Pulmonary retransplantation. J Thorac Dis 2018;10:4632-6.

20. Biswas Roy S, Panchanathan R, Walia R, et al. Lung Retransplantation for Chronic Rejection: A Single-Center Experience. Ann Thorac Surg 2018;105:221-7.

21. Verleden GM, Vos R, Verleden SE, et al. Survival determinants in lung transplant patients with chronic allograft dysfunction. Transplantation 2011;92:703-8.

22. Verleden SE, Todd JL, Sato M, et al. Impact of CLAD Phenotype on Survival After Lung Retransplantation: A Multicenter Study. Am J Transplant 2015;15:2223-30.

23. Halloran K, Aversa M, Tinckam K, et al. Comprehensive outcomes after lung retransplantation: A single-center review. Clin Transplant 2018;32:e13281.
24. Revilla-López E, Berastegui C, Sáez-Giménez B, et al. Lung Retransplantation Due to Chronic Lung Allograph Dysfunction: Results From a Spanish Transplant Unit. Archivos de Bronconeumología (English Edition) 2019;55:134-8.

25. Sato M, Waddell TK, Wagnetz U, et al. Restrictive allograft syndrome (RAS): a novel form of chronic lung allograft dysfunction. J Heart Lung Transplant 2011;30:735-42.

26. Chambers DC, Cherikh WS, Harhay MO, et al. The International Thoracic Organ Transplant Registry of the International Society for Heart and Lung Transplantation: Thirty-sixth adult lung and heart-lung transplantation Report-2019; Focus theme: Donor and recipient size match. J Heart Lung Transplant 2019;38:1042-55.

27. Eberlein M, Permutt S, Chahla MF, et al. Lung size mismatch in bilateral lung transplantation is associated with allograft function and bronchiolitis obliterans syndrome. Chest 2012;141:451-60.

28. Eberlein M, Reed RM, Bolukbas S, et al. Lung size mismatch and primary graft dysfunction after bilateral lung transplantation. J Heart Lung Transplant 2015;34:233-40.

29. Eberlein M, Reed RM, Maidaa M, et al. Donor-recipient size matching and survival after lung transplantation. A cohort study. Ann Am Thorac Soc 2013;10:418-25.

30. Massard G, Badier M, Guillot C, et al. Lung size matching for double lung transplantation based on the submammary thoracic perimeter. Accuracy and functional results. The Joint Marseille-Montreal Lung Transplant Program. J Thorac Cardiovasc Surg 1993;105:9-14.

31. Harjula A, Baldwin JC, Starnes VA, et al. Proper donor selection for heart-lung transplantation. The Stanford experience. J Thorac Cardiovasc Surg 1987;94:874-80.

32. Griffith BP, Hardesty RL, Trento A, et al. Heart-lung transplantation: lessons learned and future hopes. Ann Thorac Surg 1987;43:6-16.

33. Verleden SE, Vanaudenaerde BM, Emonds MP, et al. Donor-specific and -nonspecific HLA antibodies and outcome post lung transplantation. Eur Respir J 2017;50:1701248.

34. Kim M, Townsend KR, Wood IG, et al. Impact of pretransplant anti-HLA antibodies on outcomes in lung transplant candidates. Am J Respir Crit Care Med 2014;189:1234-9.

35. Gray AL, Mulvihill MS, Hartwig MG. Lung transplantation at Duke. J Thorac Dis 2016;8:E185-96.

36. Kulkarni HS, Bemiss BC, Hachem RR. Antibody-mediated Rejection in Lung Transplantation. Curr Transplant Rep 
2015;2:316-23.

37. Kon ZN, Bittle GJ, Pasrija C, et al. The Optimal Procedure for Retransplantation After Single Lung Transplantation. Ann Thorac Surg 2017;104:170-5.

38. Sommer W, Ius F, Kuhn C, et al. Technique and Outcomes of Less Invasive Lung Retransplantation. Transplantation 2018;102:530-7.

39. Ius F, Sommer W, Tudorache I, et al. Five-year experience with intraoperative extracorporeal membrane oxygenation

Cite this article as: Rucker AJ, Nellis JR, Klapper JA, Hartwig MG. Lung retransplantation in the modern era. J Thorac Dis 2021;13(11):6587-6593. doi: 10.21037/jtd-2021-25 in lung transplantation: Indications and midterm results. J Heart Lung Transplant 2016;35:49-58.

40. Wallinder A, Danielsson C, Magnusson J, et al. Outcomes and Long-term Survival After Pulmonary Retransplantation: A Single-Center Experience. Ann Thorac Surg 2019;108:1037-44.

41. Neuberger J, James O. Guidelines for selection of patients for liver transplantation in the era of donor-organ shortage. Lancet 1999;354:1636-9. 